\title{
The properties of kaonic nuclei in relativistic mean-field theory
}

\author{
X. H. Zhong, ${ }^{1,2 *}$ G. X. Peng, ${ }^{2 \dagger}$ L. Li, ${ }^{1}$ and P. Z. Ning, ${ }^{1,2 \ddagger}$ \\ ${ }^{1}$ Department of Physics, Nankai University, Tianjin 300071, China \\ ${ }^{2}$ Institute of High Energy Physics, Chinese Academy of Sciences, Beijing 100039, China
}

\begin{abstract}
The static properties of some possible light and moderate kaonic nuclei, from $\mathrm{C}$ to Ti, are studied in the relativistic mean-field theory. The $1 \mathrm{~s}$ and $1 \mathrm{p}$ state binding energies of $K^{-}$are in the range of $73 \sim 96 \mathrm{MeV}$ and $22 \sim 63 \mathrm{MeV}$, respectively. The binding energies of $1 \mathrm{p}$ states increase monotonically with the nucleon number A. The upper limit of the widths are about $42 \pm 14 \mathrm{MeV}$ for the $1 \mathrm{~s}$ states, and about $71 \pm 10 \mathrm{MeV}$ for the $1 \mathrm{p}$ states. The lower limit of the widths are about $12 \pm 4 \mathrm{MeV}$ for the $1 \mathrm{~s}$ states, and $21 \pm 3 \mathrm{MeV}$ for the $1 \mathrm{p}$ states. If $V_{0} \leq 30 \mathrm{MeV}$, the discrete $K^{-}$ bound states should be identified in experiment. The shrinkage effect is found in the possible kaonic nuclei. The interior nuclear density increases obviously, the densest center density is about $2.1 \rho_{0}$.
\end{abstract}

PACS numbers: 21.10.Dr, 21.30.Fe, 21.90.+f

\section{INTRODUCTION}

The $K$-nucleon $(K N)$ interactions in nuclear matter have been an interesting topic in nuclear physics, since Kaplan firstly discussed the question of kaon condensation in dense nuclear matter about twenty years ago 1]. With several typical methods, such as chiral perturbation theory (ChPT) 2, 3, 4], RMF model [3, 4, 5, 6], chiral coupled channel model [7, 8], chiral unitary model [9, 10] or other chiral model [11, 12], and the phenomenological model by fitting the $K^{-}$atomic data using a density-dependent optical potential (DD model) [13, 14], a strong attractive $K^{-}$-nucleus potential at threshold is predicted. The $K^{-}$-nucleus potential strongly depends on the model used. The DD model gives the deepest inner $K^{-}$-nucleus potential which is in the range of $150 \sim 200 \mathrm{MeV}$ 13, 14]. The chiral coupled channel model predicts the $K^{-}$-nucleus potential in the range of $85 \sim 120 \mathrm{MeV}$ [4, 6, , 8], close to that by RMF model (The $\sigma$-K coupling constant determined by the $K N$ scatering length). The chiral models give shallower $K^{-}$-nucleus potential $50 \sim 70 \mathrm{MeV}$ [4, 9, 10, 11, 12].

Based on the strong attractive $K^{-}$-nucleus potential, there maybe exist "deeply bound kaonic atoms" or kaonic nuclei. The existence of the "deeply bound kaonic atoms", or, kaonic nuclei was already well-known in later 1990s 15, 16]. It was suggested that the kaonic nuclei could be produced by the $\left(K^{-}, p\right)$ and $\left(K^{-}, n\right)$ reactions 16 . Since then, the issue on the $K^{-}$-nucleus deeply bound states (kaonic nuclei) has been a hot topic.

Theoretically, there have been many works on kaonic nuclei. The binding energies, the widths, the production methods, the nuclear densities around $K^{-}$-meson, and other aspects were discussed with different models 11,12 , 17, 18, 19, 20, 21, 22, 23, 24, 25, 26, 27]. Experimentally, some evidences for the candidate states in the $\left(K_{\text {stop }}, n\right)$ and $\left(K_{\text {stop }}, p\right)$ reactions on ${ }^{4} \mathrm{He}$ [28, 29], and in the $\left(K^{-}, n\right)$ in-flight reaction on ${ }^{16} \mathrm{O}$ [30] are reported. Recently, by using the FINUDA spectrometer installed at the $\mathrm{e}^{+} \mathrm{e}^{-}$collider DA $\Phi$ NE, the FINUDA collaboration succeeded in detecting a $K^{-}$bound state $K^{-} p p$ through its two-body decay into a $\Lambda$ hyperon and a proton [31]. However, just some months ago, Magas et al. reanalyzed the FINUDA data and claimed that the experimental spectrum can be naturally explained in their Monte Carlo simulation of the $K^{-}$absorption events in nuclei, without the need of exotic mechanisms like the formation of a $K^{-} p p$ bound state [32. Thus, further experimental and theoretical works are needed on this issue.

In the present work, we will study the static properties of the possible kaonic nuclei in the framework of relativistic mean-field theory (RMF). The pioneer works, describing the in-medium $K^{-} N$ interaction with RMF method, can be found in 5, 6, 33]. Since then, the RMF model had been widely used to describe the in-medium properties of (anti)kaon mesons [3, 4], the $K^{-}$-nucleus interaction [14], the $K^{-}$-nucleus elastic scattering [34 and the kaonic nuclei 22, 23]. In this work, the static properties of kaonic nuclei are obtained by solving, self-consistently, the equations of motion for nucleons and mesons, which are derived from the Lagrangian for nucleons and (anti)kaon mesons. To describe the widths of the $K^{-}$in nuclei, the equation of motion for $K^{-}$are modified by introducing an imaginary part of the self-energy phenomenologically. In the calculations, we will focus on the energy spectra, the nuclear density distribution, the r.m.s. radii of proton, neutron and charge distribution for the possible kaonic nuclei.

\footnotetext{
* E-mail: zhongxianhui@mail.nankai.edu.cn

$\dagger$ E-mail: gxpeng@ihep.ac.cn

‡ E-mail: ningpz@nankai.edu.cn
} 
The paper is organized as follows. In the subsequent section, the Lagrangian density is given, the equations of motion for nucleons and the meson fields $\sigma, \omega, \rho$, and Kaons are deduced, the imaginary part of the self-energies are introduced and the binding energies of $K^{-}$are defined. We then present our results and discussions of the obtained properties of kaonic nuclei in Sec. III. Finally a summary is given in Sec. IV.

\section{FORMULAS}

In relativistic mean field theory, the standard Lagrangian density for an ordinary nucleus can be written as [36, 37]

$$
\mathcal{L}_{0}=\mathcal{L}_{\text {Dirac }}+\mathcal{L}_{\sigma}+\mathcal{L}_{\omega}+\mathcal{L}_{\rho}+\mathcal{L}_{A}
$$

where

$$
\begin{aligned}
\mathcal{L}_{\text {Dirac }}= & \bar{\Psi}_{N}\left(i \gamma^{\mu} \partial_{\mu}-M_{N}\right) \Psi_{N}, \\
\mathcal{L}_{\sigma}= & \frac{1}{2} \partial_{\mu} \sigma \partial^{\mu} \sigma-\frac{1}{2} m_{\sigma}^{2} \sigma^{2}-g_{\sigma N} \bar{\Psi}_{N} \sigma \Psi_{N} \\
& -\frac{1}{3} g_{2} \sigma^{3}-\frac{1}{4} g_{3} \sigma^{4} \\
\mathcal{L}_{\omega}= & -\frac{1}{4} F_{\mu \nu} F^{\mu \nu}+\frac{1}{2} m_{\omega}^{2} \omega_{\mu} \omega^{\mu} \\
& -g_{\omega N} \bar{\Psi}_{N} \gamma^{\mu} \Psi_{N} \omega_{\mu}, \\
\mathcal{L}_{\rho}= & -\frac{1}{4} \vec{G}_{\mu \nu} \vec{G}^{\mu \nu}+\frac{1}{2} m_{\rho}^{2} \vec{\rho}_{\mu} \cdot \vec{\rho}^{\mu} \\
& -g_{\rho N} \bar{\Psi}_{N} \vec{\rho}^{\mu} \cdot \vec{I} \Psi_{N}, \\
\mathcal{L}_{A}= & -\frac{1}{4} H_{\mu \nu} H^{\mu \nu}-e \bar{\Psi}_{N} \gamma_{\mu} I_{c} A^{\mu} \Psi_{N},
\end{aligned}
$$

with

$$
\begin{aligned}
F_{\mu \nu} & =\partial_{\nu} \omega_{\mu}-\partial_{\mu} \omega_{\nu} \\
\vec{G}_{\mu \nu} & =\partial_{\nu} \vec{\rho}_{\mu}-\partial_{\mu} \vec{\rho}_{\nu} \\
H_{\mu \nu} & =\partial_{\nu} A_{\mu}-\partial_{\mu} A_{\nu}
\end{aligned}
$$

where the meson fields are denoted by $\sigma, \omega_{\mu}$, and $\vec{\rho}_{\mu}$, and their masses by $m_{\sigma}, m_{\omega}, m_{\rho}$, respectively. $\Psi_{N}$ is the nucleon field with corresponding mass $M_{N}$. $A_{\mu}$ is the electromagnetic field. $g_{\sigma N}, g_{\omega N}$, and $g_{\rho N}$ are, respectively, the $\sigma-N, \omega-N$, and $\rho-N$ coupling constants. $I_{c}=\left(1+\tau_{3}\right) / 2$ is the Coulomb interaction operator with $\tau_{3}$ being the third component of the isospin Pauli matrices for nucleons. $I$ is the nucleon isospin operator. In this paper, we adopt the NL-SH parameter set 38], which describes the properties of finite nuclei reasonably. The masses and coupling constants are listed in Tab. [1]

For a $K^{-}$-nucleus system, another Lagrangian density $\mathcal{L}_{\mathrm{K}}$ describing the (anti)kaon interaction with nucleons should be added to $\mathcal{L}_{0}$. Kaons are incorporated into the RMF model by using the $K N$ interactions motivated by the one-meson-exchange models [35]. In the meson-exchange picture, the scalar and vector interactions between kaons and nucleons are mediated by the exchange of $\sigma$ and $\omega$ mesons, respectively. The coupling of the (anti)kaon to the isovector $\rho$ meson is here excluded due to $N=Z$ nuclear cores. Thus, the simplest kaon-meson interaction Lagrangian density $\mathcal{L}_{\mathrm{K}}$ is written as $[6]$

$$
\begin{aligned}
\mathcal{L}_{\mathrm{K}}= & \partial_{\mu} \bar{K} \partial^{\mu} K-m_{K}^{2} \bar{K} K-g_{\sigma K} m_{K} \bar{K} K \sigma \\
& -i g_{\omega K}\left(\bar{K} \partial_{\mu} K-K \partial_{\mu} \bar{K}\right) \omega^{\mu} \\
& +\left(g_{\omega K} \omega^{\mu}\right)^{2} \bar{K} K
\end{aligned}
$$

where $g_{\sigma \mathrm{K}}$ and $g_{\omega \mathrm{K}}$ are the $\sigma-K$ and $\omega-K$ coupling constants. $g_{\omega \mathrm{K}}$ is chosen from the SU(3) relation assuming ideal mixing, i.e., $2 g_{\omega \mathrm{K}}=2 g_{\pi \pi \rho}=6.04$. $g_{\sigma \mathrm{K}}$ can be obtained from several methods, e.g. the Bonn model [35], by reproducing the strongly attractive potential seen in kaonic atoms 14] or by fitting the $K N$ scattering lengths in experiments [4, 6]. In this paper, we take the modest $K-\sigma$ coupling, $g_{\sigma K}=2.088\left(\approx g_{\sigma N} / 5\right)$, by fitting the experimental $K N$ scattering length 4]. The advantages of this method were pointed out in 4]. With these determined coupling constants, the antikaon optical potential at normal nuclear density is about $U_{\mathrm{K}^{-}}=-(85 \sim 100) \mathrm{MeV}[\underline{6}]$, which is 
compatible with several groups' predictions. For example, Akaishi et al. gave $-119 \mathrm{MeV}$ for a $K^{-}$in nuclear matter at the normal density [19], and Weise's gave $U_{\mathrm{K}^{-}}=-(120 \sim 130) \mathrm{MeV}$ by using chiral $K N$ interaction [7]. It is interesting that the recent experiment predicts the in-medium $K^{-} N$ potential is on the order of $-80 \mathrm{MeV}$ at normal nuclear density [4], which is also agreement with the $K^{-} N$ potential adopted in present paper.

TABLE I: Parameters used in the present calculations.

\begin{tabular}{|c|c|c|c|c|c|c|c|}
\hline \multicolumn{4}{|c|}{ masses $(\mathrm{MeV})$} & \multicolumn{4}{|c|}{ couplings } \\
\hline$M_{N}$ & $m_{\sigma}$ & $m_{\omega}$ & $m_{\rho}$ & $g_{\sigma N}$ & $g_{\omega N}$ & $g_{\rho N}$ & $g_{3}$ \\
\hline 939.0 & 526.059 & 783.0 & 763.0 & $\begin{array}{r}10.444 \\
g\end{array}$ & $\begin{array}{c}12.94 \\
=-6\end{array}$ & $\begin{array}{l}8.766 \\
9099\end{array}$ & $\begin{array}{l}5.8337 \\
1\end{array}$ \\
\hline
\end{tabular}

In the mean field approximation, the meson-fields $\sigma, \omega_{\mu}$, and $\rho_{\mu}$, and the photons $A_{\mu}$ are replaced with their mean values, $\langle\sigma\rangle,\left\langle\omega_{\mu}\right\rangle,\left\langle\rho_{\mu}\right\rangle$ and $\left\langle A_{\mu}\right\rangle$, respectively. For a spherical nucleus, only the mean values of the time components $\left\langle\omega_{0}\right\rangle,\left\langle\rho_{0}\right\rangle$ and $\left\langle A_{0}\right\rangle$ remain, which are denoted by $\omega_{0}$, and $\rho_{0}$, and $A_{0}$ respectively. Then the equations of motion for nucleons, $\omega, \sigma, \rho$, and photons are

$$
\begin{gathered}
{\left[-i \vec{\alpha} \cdot \vec{\nabla}+\beta\left(M_{N}+g_{\sigma N} \sigma_{0}\right)+g_{\omega N} \omega_{0}+g_{\rho N} \tau_{3} \rho_{0}+e I_{c} A_{0}\right] \Psi_{N}=\epsilon \Psi_{N}} \\
\left(-\nabla^{2}+m_{\sigma}^{2}\right) \sigma_{0}=-g_{\sigma N} \bar{\Psi}_{N} \Psi_{N}-g_{2} \sigma_{0}^{2}-g_{3} \sigma_{0}^{3}-g_{\sigma K} m_{K} \bar{K} K \\
\left(-\nabla^{2}+m_{\omega}^{2}\right) \omega_{0}=g_{\omega N} \bar{\Psi}_{N} \gamma^{0} \Psi_{N}-2 g_{\omega K}\left(E+g_{\omega K} \omega_{0}\right) \bar{K} K \\
\left(-\nabla^{2}+m_{\rho}^{2}\right) \rho_{0}=g_{\rho N} \bar{\Psi}_{N} \gamma^{0} I \Psi_{N} \\
-\nabla^{2} A_{0}=e \bar{\Psi}_{N} \gamma^{0} I_{c} \Psi_{N}
\end{gathered}
$$

and the equation of motion for antikaon is

$$
\left[-\nabla^{2}+\left(m_{\mathrm{K}}^{2}-E^{2}\right)+\Pi\right] \bar{K}=0,
$$

with the antikaon self-energy in nuclei

$$
\Pi=-2 g_{\omega \mathrm{K}} E \omega_{0}+g_{\sigma \mathrm{K}} m_{\mathrm{K}} \sigma_{0}-\left(g_{\omega \mathrm{K}} \omega_{0}\right)^{2} .
$$

In the above equations, $\epsilon$ is the nucleon single-particle energy, and $E$ is the single-particle energy for antikaon meson. We can see that the antikaon meson does not relate the Dirac equation for nucleons directly. However, the presence of antikaon leads to additional source terms in the equations of motion for meson fields of $\sigma$ and $\omega$. Thus, the antikaon meson affects the nucleon fields by changing the strength of meson fields of $\sigma$ and $\omega$ indirectly.

So far, we ignored the antikaon meson absorption in the nucleus, which requires a complex potential. Within the framework of RMF model, we are not able to calculate the imaginary part of the potential directly. In order to include the effects of the antikaon meson absorption in the nucleus on the calculations, and make a more realistic estimate for the calculated results, we assume a specific form for the antikaon self-energy with an imaginary part

$$
\begin{aligned}
\widetilde{\Pi}= & {\left[-2 g_{\omega \mathrm{K}} \omega_{0} \operatorname{Re} E+g_{\sigma \mathrm{K}} m_{\mathrm{K}} \sigma_{0}-\left(g_{\omega \mathrm{K}} \omega_{0}\right)^{2}\right] } \\
& +i\left[-2(\operatorname{Re} E) f V_{0} \frac{\rho}{\rho_{0}}\right] .
\end{aligned}
$$

The similar method is also used to analyze the widths of mesonic nuclei in [22, 44].

In this work, the imaginary part of the potential $\operatorname{Im} U$ is given with the simple " $t \rho$ " form, namely, $\operatorname{Im} U=-V_{0} \rho / \rho_{0}$. Where $V_{0}$ is the imaginary potential depth at normal nuclear density, which strongly depends on the model adopted. The largest value $V_{0} \sim 50 \mathrm{MeV}$ is given by fitting the experimental data of the kaonic atoms [13, 14], and the chiral model (only the antikaons are dressed self-consistently) 45]. While, by fitting the experimental data of the antikaonnucleus scattering, the imaginary potential depth become much shallower: $V_{0} \sim 35 \mathrm{MeV}[34$. Another much shallower imaginary potential depth $20 \sim 25 \mathrm{MeV}$ is predicted by Ramos et al. with the meson-exchange model. The shallowest imaginary potential depth, $\sim 15 \mathrm{MeV}$, is given by the chiral model which incorporates the dressing of the pion and antikaons [4]. Thus, in the present work, we set the imaginary potential depth $V_{0}$ in the range of $15 \sim 50 \mathrm{MeV}$.

On the other hand, the phase space available for the decay products should be reduced for deeply bound states, which will decrease the imaginary potentials (widths). Thus, a suppression factor, $f$, multiplying Im $U$ were suggested to be introduced by Mareš et al. [22, 23]. In our calculations, we adopt their method as well. In this method, two decay channels are considered. One is the mesonic decay channel, $\bar{K} N \rightarrow \pi \Sigma$, $\pi \Lambda$. The corresponding suppression factor is given by 


$$
f_{1}=\frac{M_{01}^{3}}{M_{1}^{3}} \sqrt{\frac{\left[M_{1}^{2}-\left(m_{\pi}+M_{Y}\right)^{2}\right]\left[M_{1}^{2}-\left(m_{\pi}-M_{Y}\right)^{2}\right]}{\left[M_{01}^{2}-\left(m_{\pi}+M_{Y}\right)^{2}\right]\left[M_{01}^{2}-\left(m_{\pi}-M_{Y}\right)^{2}\right]}} \Theta\left(M 1-m_{\pi}-M_{Y}\right),
$$

where $M_{01}=m_{k}+M_{N}, M_{1}=\operatorname{Re} E+M_{N}$ and $Y=\Sigma, \Lambda$. The other channel is the non-mesonic decay channel, $\bar{K} N N \rightarrow Y N$, and the corresponding suppression factor is

$$
f_{2}=\frac{M_{02}^{3}}{M_{2}^{3}} \sqrt{\frac{\left[M_{2}^{2}-\left(M_{N}+M_{Y}\right)^{2}\right]\left[M_{2}^{2}-\left(M_{N}-M_{Y}\right)^{2}\right]}{\left[M_{02}^{2}-\left(M_{N}+M_{Y}\right)^{2}\right]\left[M_{02}^{2}-\left(M_{N}-M_{Y}\right)^{2}\right]}} \Theta\left(M_{2}-M_{N}-M_{Y}\right),
$$

where $M_{02}=m_{k}+2 M_{N}, M_{2}=\operatorname{Re} E+2 M_{N}$. Since $\Sigma$ final states dominate both the mesonic and non-mesonic decay channels [46], in the calculations, the hyperon $Y$ is set as $Y=\Sigma$. The suppression factor $f$ can be assumed a mixture of $80 \%$ mesonic decay and $20 \%$ non-mesonic decay [46], thus

$$
f=0.8 f_{1}+0.2 f_{2} \text {. }
$$

In the calculations, in order to include the effects from the "imaginary potential", we use the modified Klein-Gordon equation

$$
\left[-\nabla^{2}+\left(m_{\mathrm{K}}^{2}-E^{2}\right)+\widetilde{\Pi}\right] \bar{K}=0
$$

The complex eigenenergies are

$$
E=-B_{K}^{s, p}+m_{K}-i \Gamma / 2,
$$

where the real part corresponds to the single-particle $K^{-}$binding energy, which is defined as

$$
B_{\mathrm{K}}^{s, p}=m_{\mathrm{K}}-\operatorname{Re} E,
$$

and the imaginary part of the complex eigenenergies corresponds to the widths

$$
\Gamma=-2 \operatorname{Im} E .
$$

The binding energy of $K^{-}, B_{K^{-}}$, is defined as the difference between the total binding energy of the $K^{-}$-nucleus system, $B\left({ }^{A} Z K^{-}\right)$, and the total binding energy of the ordinary nucleus, $B\left({ }^{A} Z\right)[23]$ :

$$
B_{\mathrm{K}}=B\left({ }^{A} Z K^{-}\right)-B\left({ }^{A} Z\right) .
$$

In RMF, from the Lagrangian density (Eqs.(1-6)) we can deduce the energy-momentum tensor $T^{\mu \nu}$ and then obtain the total binding energy of the ordinary nucleus at once:

$$
\begin{aligned}
B\left({ }^{A} Z\right)= & A \times M_{N}+E_{c . m .}-\sum_{i=1}^{A} \epsilon_{i} \\
& -\frac{1}{2} \int d \mathbf{r}\left\{-g_{\sigma N} \sigma_{0} \bar{\Psi}_{N} \Psi_{N}-g_{2} \sigma_{0}^{2}\right. \\
& -g_{3} \sigma_{0}^{3}-g_{\omega N} \omega_{0} \Psi_{N}^{\dagger} \Psi_{N} \\
& \left.-g_{\rho N} \bar{\Psi}_{N} \gamma^{0} I \Psi_{N}-e \bar{\Psi}_{N} \gamma^{0} I_{c} \Psi_{N}\right\},
\end{aligned}
$$

where $\epsilon_{i}$ is the nucleon single-particle energy labelled $i$, and $E_{c . m .}=3 / 4 \cdot 41 A^{1 / 3}$ is the center-of-mass energy. Using the same method, according to the Eqs. $(1-6)$ and (10) we obtain the total binding energy of the $K^{-}$-nucleus system:

$$
\begin{aligned}
B\left({ }^{A} Z K^{-}\right)= & A \times M_{N}+E_{c . m .}+B_{\mathrm{K}}^{s, p} \\
& -\sum_{i=1}^{A} \epsilon_{i}-\frac{1}{2} \int d \mathbf{r}\left\{-g_{\sigma N} \sigma_{0} \bar{\Psi}_{N} \Psi_{N}\right. \\
& -g_{2} \sigma_{0}^{2}-g_{3} \sigma_{0}^{3}-g_{\omega N} \omega_{0} \Psi_{N}^{\dagger} \Psi_{N} \\
& -g_{\rho N} \bar{\Psi}_{N} \gamma^{0} I \Psi_{N}-e \bar{\Psi}_{N} \gamma^{0} I_{c} \Psi_{N} \\
& +2 g_{\omega K} \omega_{0}\left(3 \operatorname{Re} E+g_{\omega K} \omega_{0}\right) \bar{K} K \\
& \left.-g_{\sigma K} m_{K} \sigma_{0} \bar{K} K\right\} .
\end{aligned}
$$


From the above, we can see that for the appearance of the antikaon there are additional terms, $-g_{\sigma K} m_{K} \sigma_{0} \bar{K} K$ and $2 g_{\omega K}\left(3 \operatorname{Re} E+g_{\omega K} \omega_{0}\right) \omega_{0}$, in the integral of Eq.(28) compared with those of Eq. (27).

Solving the equations (11) - (15) and Eq. 222) self-consistently, we can obtain the properties of $K^{-}$-nuclei. One point must be noted that the antikaon energy $E$ in Eq.(13) should be replaced with its real part, ReE, in the calculations.

\section{RESULTS AND DISCUSSIONS}

The main purpose of the present calculations is to obtain the properties of $K^{-}$nuclei in RMF, such as the binding energies, the nuclear density distributions and the r.m.s. radii of proton, neutron and charge distributions. We carry out calculations for the $N=Z$ even-even nuclear cores ${ }^{12} \mathrm{C} K^{-},{ }^{16} \mathrm{O} K^{-},{ }^{20} \mathrm{Ne} K^{-},{ }^{24} \mathrm{Mg} K^{-},{ }^{28} \mathrm{Si} K^{-},{ }^{32} \mathrm{~S} K^{-}$, ${ }^{36} \mathrm{Ar} K^{-},{ }^{40} \mathrm{Ca} K^{-}$and ${ }^{44} \mathrm{Ti} K^{-}$, respectively. For the imaginary potential depths at normal nuclear density are not well determined, in the calculations, we choose three values, $V_{0}=15,30$ and $50 \mathrm{MeV}$, respectively. The results are shown in Tab. II III and Figs. 1

TABLE II: The single-particle $K^{-}$binding energies, $B_{K^{-}}^{s, p}=m_{K}-R e E$; the binding energy of $K^{-}, B_{K^{-}}$; the total binding energy of the $K^{-}$-nucleus system, $B\left({ }^{A} Z K^{-}\right)$; the total binding energy of the ordinary nucleus, $B\left({ }^{A} Z\right)$ and the widths, $\Gamma$, (all in $\mathrm{MeV}$ ), in various nuclei, where the complex eigenenergies are, $E=-B_{K}^{s, p}+m_{K}-i \Gamma / 2$.

\begin{tabular}{|c|c|c|c|c|c|c|c|c|c|c|c|c|c|c|}
\hline & \multicolumn{5}{|c|}{$\underline{V_{0}=15(\mathrm{MeV})}$} & \multicolumn{4}{|c|}{$V_{0}=30(\mathrm{MeV})$} & \multicolumn{4}{|c|}{$\underline{V_{0}=50(\mathrm{MeV})}$} \\
\hline & & $B\left({ }^{A} Z\right)$ & $B\left({ }^{A} \overline{Z K^{-}}\right)$ & $B_{K}$ & $B_{K}^{s, p}$ & $\Gamma$ & $B\left({ }^{A} \overline{\left.Z K^{-}\right)}\right.$ & $B_{K}$ & $B_{K}^{s, p}$ & $\Gamma$ & $B\left({ }^{A} \overline{\left.Z K^{-}\right)}\right.$ & $B_{K}$ & $B_{K}^{s, p}$ & $\Gamma$ \\
\hline \multirow[t]{2}{*}{${ }^{12} \mathrm{C} K^{-}$} & $1 s$ & 89.6 & 177.6 & 88.0 & 102.3 & 11.2 & 177.0 & 87.4 & 100.4 & 19.4 & 175.6 & 86.0 & 97.6 & 44.0 \\
\hline & $1 p$ & & 115.2 & 25.6 & 35.1 & 23.5 & 114.5 & 24.8 & 33.2 & 47.0 & 111.2 & 21.6 & 28.8 & 80.9 \\
\hline \multirow[t]{2}{*}{${ }^{16} \mathrm{O} K^{-}$} & $1 s$ & 128.5 & 203.8 & 75.3 & 88.3 & 16.2 & 203.4 & 74.8 & 86.7 & 32.8 & 202.4 & 73.9 & 84.4 & 56.4 \\
\hline & $1 p$ & & 158.7 & 30.2 & 41.1 & 21.1 & 157.7 & 29.2 & 38.5 & 43.0 & 156.9 & 28.4 & 36.6 & 72.8 \\
\hline \multirow[t]{2}{*}{${ }^{20} \mathrm{Ne} K^{-}$} & $1 s$ & 142.6 & 221.0 & 78.4 & 90.4 & 14.8 & 220.8 & 78.2 & 89.4 & 30.0 & 220.0 & 77.4 & 87.7 & 51.4 \\
\hline & $1 p$ & & 180.2 & 37.7 & 48.9 & 21.5 & 179.9 & 37.3 & 47.5 & 42.8 & 178.5 & 35.9 & 45.3 & 71.5 \\
\hline \multirow{2}{*}{${ }^{24} \mathrm{Mg} K^{-}$} & $1 s$ & 182.8 & 267.5 & 84.7 & 95.3 & 12.2 & 267.4 & 84.6 & 94.7 & 25.2 & 266.8 & 84.0 & 93.3 & 43.6 \\
\hline & $1 p$ & & 231.0 & 48.2 & 59.4 & 22.0 & 230.7 & 47.9 & 58.5 & 42.6 & 229.1 & 46.3 & 56.4 & 74.0 \\
\hline \multirow[t]{2}{*}{${ }^{28} \mathrm{Si} K^{-}$} & $1 s$ & 231.6 & 322.4 & 90.8 & 100.2 & 8.2 & 322.2 & 90.6 & 99.6 & 17.8 & 321.8 & 90.2 & 98.7 & 32.6 \\
\hline & $1 p$ & & 289.0 & 56.9 & 68.4 & 20.3 & 288.2 & 56.6 & 67.2 & 40.7 & 287.0 & 55.4 & 65.7 & 68.6 \\
\hline \multirow[t]{2}{*}{${ }^{32} \mathrm{~S} K^{-}$} & $1 s$ & 261.6 & 358.1 & 96.5 & 107.8 & 14.2 & 358.1 & 96.5 & 106.8 & 27.0 & 357.8 & 96.2 & 104.1 & 37.0 \\
\hline & $1 p$ & & 320.1 & 58.5 & 67.5 & 20.2 & 319.8 & 58.2 & 66.9 & 40.6 & 317.3 & 55.7 & 66.3 & 68.8 \\
\hline \multirow[t]{2}{*}{${ }^{36} \mathrm{Ar} K^{-}$} & $1 s$ & 295.2 & 387.8 & 92.6 & 102.4 & 9.2 & 387.7 & 92.5 & 101.3 & 14.4 & 387.3 & 92.1 & 100.0 & 27.8 \\
\hline & $1 p$ & & 352.9 & 57.7 & 68.1 & 19.2 & 352.6 & 57.4 & 67.4 & 39.1 & 351.6 & 56.3 & 66.1 & 66.0 \\
\hline \multirow[t]{2}{*}{${ }^{40} \mathrm{CaK}{ }^{-}$} & $1 s$ & 340.6 & 430.4 & 89.8 & 99.0 & 9.3 & 430.3 & 89.7 & 98.0 & 20.1 & 429.7 & 89.2 & 96.4 & 36.3 \\
\hline & $1 p$ & & 400.6 & 60.0 & 69.3 & 18.6 & 400.4 & 59.8 & 68.7 & 37.3 & 399.5 & 59.0 & 67.6 & 62.9 \\
\hline \multirow[t]{2}{*}{${ }^{44} \mathrm{Ti} K^{-}$} & $1 s$ & 365.6 & 455.8 & 90.3 & 98.5 & 9.4 & 455.5 & 90.0 & 97.7 & 19.9 & 454.7 & 89.2 & 96.3 & 35.7 \\
\hline & $1 p$ & & 428.8 & 63.6 & 72.3 & 18.0 & 428.5 & 63.0 & 71.7 & 36.1 & 427.6 & 62.1 & 70.7 & 61.0 \\
\hline
\end{tabular}

\section{A. Single-particle energies and widths}

The single-particle $K^{-}$binding energies, $B_{K^{-}}^{s, p}$, the binding energy of $K^{-}, B_{K^{-}}$, the total binding energy of the kaonic nucleus, $B\left({ }^{A} Z K^{-}\right)$, the total binding energy of the ordinary nucleus, $B\left({ }^{A} Z\right)$ and the widths, $\Gamma$ are listed in Tab. II From the Tab. we find that the imaginary potentials (namely, the width) have a few effects on the values of $B_{K^{-}}^{s, p}, B_{K^{-}}$and $B\left({ }^{A} Z K^{-}\right)$. These values decrease with the increment of the imaginary potential depth. 
The decreased values are about $1 \sim 2 \mathrm{MeV}$, if $V_{0}$ changes from $15 \mathrm{MeV}$ to $50 \mathrm{MeV}$. The single-particle $K^{-}$binding energies of $1 \mathrm{~s}$ states are about $7 \sim 12 \mathrm{MeV}$ larger than the $K^{-}$binding energies of 1 s states, $B_{K^{-}}^{1 s}$. On the other hand, the single-particle $K^{-}$binding energies of $1 \mathrm{p}$ states are about $7 \sim 10 \mathrm{MeV}$ larger than the $K^{-}$binding energies of $1 \mathrm{p}$ states, $B_{K^{-}}^{1 p}$. Mareš et al. also predicted $B_{K^{-}}^{s, p}>B_{K^{-}}$in their calculations, they defined the difference as "rearrangement energy", which relates the polarization of the nuclear core by the $K^{-}$. The rearrangement energies of 1 s states decrease monotonically with the nucleon number as a whole in our calculations.

The $1 \mathrm{~s}$ state binding energies of $K^{-}, B_{K^{-}}^{1 s}$, for the listed nuclei are in the range of $B_{K^{-}}^{1 s}=73 \sim 96 \mathrm{MeV}$. And the binding energies of $K^{-}$for $1 \mathrm{p}$ states, $B_{K^{-}}^{1 p}$, range from $22 \mathrm{MeV}$ to $63 \mathrm{MeV}$, increase monotonically with the nucleon number $A$. Recently the FINUDA experiment predicted there is a $K^{-} p p$ cluster with binding energy $B_{\mathrm{Kpp}} \sim 115$ $\mathrm{MeV}[31]$, subtracting the binding energy between the two protons, $B_{p p}=27.2 \mathrm{MeV}$, the $K^{-}$binding energy is $\sim 88$ $\mathrm{MeV}$. Our predictions $(73 \sim 96 \mathrm{MeV})$ are compatible with the FINUDA experimental values and the theoretical calculations in [19, 20].

The separations between the bound state $1 \mathrm{p}$ and $1 \mathrm{~s},\left(B_{K^{-}}^{1 s}-B_{K^{-}}^{1 p}\right)$, are on the order of $27 \sim 62 \mathrm{MeV}$, decrease with the increment of the nucleon number in general. Increasing the imaginary potential depth from $V_{0}=15 \mathrm{MeV}$ to $50 \mathrm{MeV}$, the separations between the $1 \mathrm{p}$ and $1 \mathrm{~s}$ states will increase $\sim 1 \mathrm{MeV}$.

On the other hand, from Tab. II we find that the widths of the 1s kaonic bound states are about $12 \pm 4 \mathrm{MeV}, 23 \pm 9$ $\mathrm{MeV}$ and $42 \pm 14 \mathrm{MeV}$ for $V_{0}=15,30$ and $50 \mathrm{MeV}$, respectively. The $1 \mathrm{p}$ state widths are on the order of 20, 40, for $V_{0}=15$ and $30 \mathrm{MeV}$, respectively. And for $V_{0}=50 \mathrm{MeV}$, the $1 \mathrm{p}$ state widths are about $60 \sim 80 \mathrm{MeV}$, decrease with the increment of the nucleon number in general. Mareš et al. analyzed the FINUDA experiment, considering the effects of Fermi-motion on the decay widths the $K^{-} p p$ decay width $\Gamma \sim 67 \mathrm{MeV}$ will be reduced down to $53 \mathrm{MeV}$. This result agrees with our predictions $\Gamma^{1 s}=42 \pm 14 \mathrm{MeV}$ with $V_{0}=50 \mathrm{MeV}$.

From Tab. [II we can also see that, if the depth $V_{0} \leq 30 \mathrm{MeV}$, the sum of the half widths of the $1 \mathrm{~s}$ and $1 \mathrm{p}$ states predicted by us are narrower than the separations between $B_{K^{-}}^{1 s}$ and $B_{K^{-}}^{1 p}$, which implies that some discrete states should be identified in experiment for these nuclei. However, if $V_{0} \geq 50 \mathrm{MeV}$ the sum of the half widths of the $1 \mathrm{~s}$ and $1 \mathrm{p}$ states for all the listed nuclei, except ${ }^{12} \mathrm{C} K^{-}$, predicted by us are larger than the separations of the two lowest energy levels, thus no discrete states can be identified in experiment.

TABLE III: The r.m.s. radii of neutron, proton and charge distributions, $r_{n}, r_{p}$ and $r_{c h}$ (in fm), respectively. $V_{0}$ (in MeV) is the imaginary potential depth at normal nuclear density.

\begin{tabular}{|c|c|c|c|c|c|c|c|c|c|c|c|c|c|c|}
\hline & $V_{0}$ & $r_{p}$ & $r_{n}$ & $r_{c h}$ & & $V_{0}$ & $r_{p}$ & $r_{n}$ & $r_{c h}$ & & $V_{0}$ & $r_{p}$ & $r_{n}$ & $r_{c h}$ \\
\hline${ }^{12} \mathrm{C}$ & & 2.32 & 2.30 & 2.46 & ${ }^{24} \mathrm{Mg}$ & & 2.86 & 2.82 & 2.97 & ${ }^{36} \mathrm{Ar}$ & & 3.26 & 3.21 & 3.36 \\
\hline \multirow{3}{*}{${ }^{12} \mathrm{C} K^{-}$} & 15 & 2.20 & 2.18 & 2.35 & ${ }^{24} \mathrm{Mg} K^{-}$ & 15 & 2.80 & 2.77 & 2.92 & ${ }^{36} \operatorname{Ar} K^{-}$ & 15 & 3.22 & 3.17 & 3.32 \\
\hline & 30 & 2.20 & 2.18 & 2.35 & & 30 & 2.80 & 2.77 & 2.92 & & 30 & 3.22 & 3.17 & 3.32 \\
\hline & 50 & 2.21 & 2.19 & 2.35 & & 50 & 2.80 & 2.77 & 2.92 & & 50 & 3.22 & 3.18 & 3.33 \\
\hline${ }^{16} \mathrm{O}$ & & 2.58 & 2.55 & 2.70 & ${ }^{28} \mathrm{Si}$ & & 2.93 & 2.90 & 3.04 & ${ }^{40} \mathrm{Ca}$ & & 3.36 & 3.31 & 3.46 \\
\hline \multirow[t]{3}{*}{${ }^{16} \mathrm{O} K^{-}$} & 15 & 2.52 & 2.49 & 2.65 & ${ }^{28} \mathrm{Si} K^{-}$ & 15 & 2.88 & 2.85 & 2.99 & ${ }^{40} \mathrm{Ca} K^{-}$ & 15 & 3.32 & 3.27 & 3.42 \\
\hline & 30 & 2.51 & 2.49 & 2.64 & & 30 & 2.88 & 2.85 & 2.99 & & 30 & 3.32 & 3.28 & 3.42 \\
\hline & 50 & 2.51 & 2.48 & 2.64 & & 50 & 2.88 & 2.85 & 2.99 & & 50 & 3.32 & 3.28 & 3.43 \\
\hline${ }^{20} \mathrm{Ne}$ & & 2.82 & 2.74 & 2.94 & ${ }^{32} \mathrm{~S}$ & & 3.13 & 3.09 & 3.24 & ${ }^{44} \mathrm{Ti}$ & & 3.44 & 3.39 & 3.54 \\
\hline \multirow[t]{3}{*}{${ }^{20} \mathrm{Ne} K^{-}$} & 15 & 2.77 & 2.69 & 2.89 & ${ }^{32} \mathrm{~S} K^{-}$ & 15 & 3.08 & 3.04 & 3.19 & ${ }^{44} \mathrm{Ti} K^{-}$ & 15 & 3.41 & 3.36 & 3.50 \\
\hline & 30 & 2.77 & 2.68 & 2.89 & & 30 & 3.08 & 3.04 & 3.19 & & 30 & 3.41 & 3.36 & 3.50 \\
\hline & 50 & 2.76 & 2.68 & 2.88 & & 50 & 3.09 & 3.04 & 3.20 & & 50 & 3.41 & 3.36 & 3.51 \\
\hline
\end{tabular}

\section{B. The static properties of the kaonic nuclei}

First, let's see Tab. III] We find that the r.m.s. radii of neutron, proton and charge distributions, $r_{n}, r_{p}$ and $r_{c h}$ for the kaonic nuclei, ${ }^{A} Z K^{-}$, are smaller than those for the corresponding ordinary nuclei, ${ }^{A} Z$. For example, the r.m.s. radii of ${ }^{12} \mathrm{C} K^{-}$reduce about $0.12 \mathrm{fm}$, when a $K^{-}$-meson is injected into ${ }^{12} \mathrm{C}$. For the other heavier kaonic nuclei, the decreased values of the r.m.s. radii are about $0.04 \sim 0.06 \mathrm{fm}$. Generally, the r.m.s. radii of lighter kaonic nuclei decrease more obviously than those of heavier kaonic nuclei. The phenomenon of the r.m.s. radii decreased 
because of a meson or hyperon being bound in a nucleus is called "shrinkage", which has been found in some lighter $\Lambda$ hypernuclei 40 in experiment. Within the framework of RMF, we also find the shrinkage effects exist in $\Lambda_{-}^{-}, \Theta^{+}-$ hypernuclei 41, 42].

From the table, we also find that the r.m.s. radii do not change obviously with the imaginary potential depth. If there are differences between the r.m.s. radii for different imaginary potential depth, the differences are within 0.01 $\mathrm{fm}$.

To explain the "shrinkage effect", we should start with the $K^{-}$properties in nuclear matter and the equations of motion for kaonic nuclei in RMF theory. Firstly, the $K^{-}$meson does not identify with nucleons and can enter the nuclear cores. Secondly, the strong $K^{-} N$ attraction can produce a strong $K$ field in nuclei, which increases the strength of the scalar field $\sigma$ (see Eq. (12)) and decreases the vector field $\omega$ [see Eq. (13)]. This means the attraction between nucleons become larger and the repulse between the nucleons become weaker. Thus, the nucleons in the nucleus are bounded more tightly, which results in the so called "shrinkage effect".

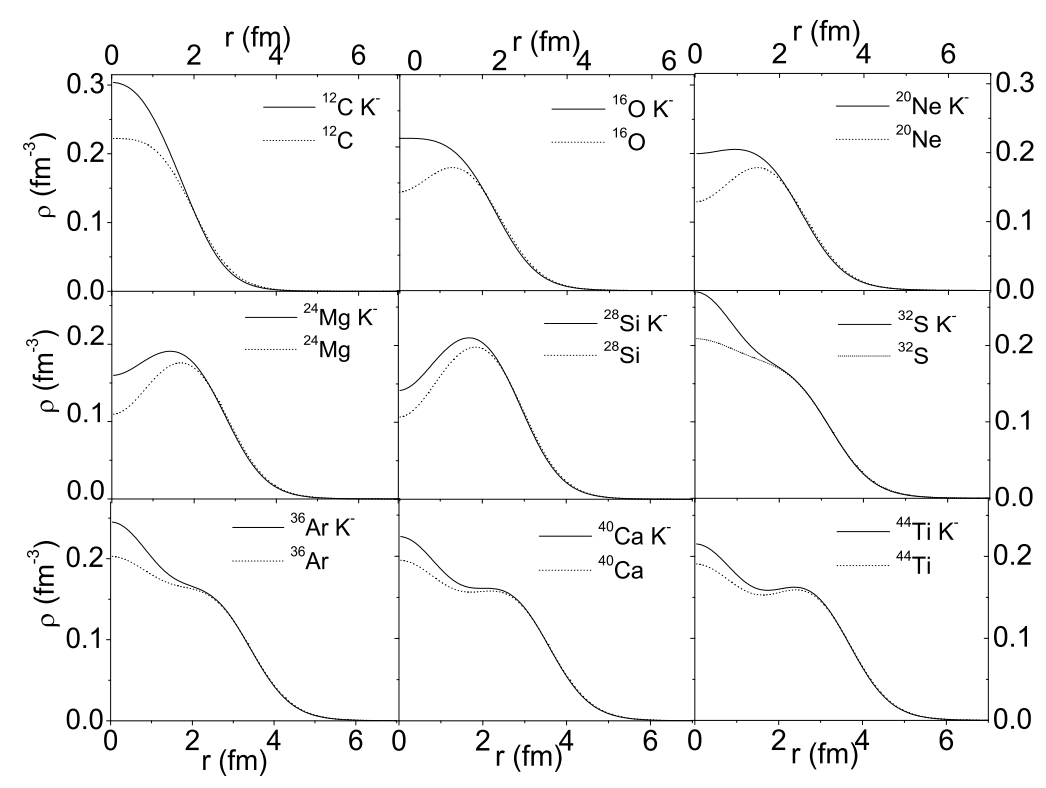

FIG. 1: Nucleon-density as a function of nucleus radius. The solid and dotted curves are for kaonic nuclei and corresponding ordinary nuclei, respectively.

\section{C. nuclear density of the kaonic nuclei}

The distributions of the nuclear density for some light and moderate kaonic nuclei in their ground states together with those of corresponding ordinary nuclei are shown in Fig. 1 In the calculations, we set $V_{0}=30 \mathrm{MeV}$.

From the figure we can see, in the interior of the kaonic nuclei, the nuclear densities become much denser than those of the ordinary nuclei. The central nuclear density is enhanced by a value $0.03 \sim 0.1 \mathrm{fm}^{-3}$ for all the listed kaonic nuclei. In all the kaonic nuclei, the lowest enhanced central nuclear density is $\sim 0.03 \mathrm{fm}^{-3}$ for ${ }^{44} \mathrm{Ti} K^{-}$, and the highest enhanced central nuclear density is $\sim 0.1 \mathrm{fm}^{-3}$ for ${ }^{12} \mathrm{C} K^{-}$. In all the listed kaonic nuclei, the central nuclear density of ${ }^{12} \mathrm{CK}^{-}$is the densest, $\sim 0.31 \mathrm{fm}^{-3}$, which is around 2.1 times the normal nuclear density $\rho_{0}$.

Our calculations give obvious enhancement of the nuclear density in the interior of the kaonic nuclei. However, this value is much less than the prediction in [19]. In [19], Akaishi et al. gave the central density about 3 times that of the $\alpha$ particle for ${ }^{8} \mathrm{Be} K^{-}$system, and in [20], the central nuclear density of kaonic nuclei gave by Doté et al. even reaches to $0.71 \sim 1.50 \mathrm{fm}^{-3}$, which is about $4 \sim 10$ times the normal nuclear density with a framework of antisymmetrized molecular dynamics. It is indeed an amazing high central density $\rho(0)=0.81 \mathrm{fm}^{-3} \sim 5 \rho_{0}$ for the many-body system ${ }^{11} \mathrm{CK}^{-}$in [20] compared with our results. 


\section{SUMMARY}

We study the kaonic nuclei within the framework of RMF theory. All the equations are solved self-consistently. We carry out systematic calculations for the light and moderate possible kaonic nuclei, from ${ }^{12} \mathrm{C}$ to ${ }^{44} \mathrm{Ti}$. The energy spectra of these kaonic nuclei are studied, the ground state (1s state) binding energies of $K^{-}$are obtained, which are in the range of $73 \sim 96 \mathrm{MeV}$ for all the possible kaonic nuclei studied. The binding energies of of $K^{-}$for $1 \mathrm{p}$ states are in the range of $22 \sim 63 \mathrm{MeV}$, and increase monotonically with the nucleon number $A$. The separations between the bound state $1 \mathrm{p}$ and $1 \mathrm{~s}$ are in the range of $27 \sim 62 \mathrm{MeV}$, and decrease with the increment of the nucleon number generally.

The upper limit widths of the $1 \mathrm{~s}$ and $1 \mathrm{p}$ states are $42 \pm 14 \mathrm{MeV}$ and $71 \pm 10 \mathrm{MeV}$, respectively, with $V_{0} \sim 50 \mathrm{MeV}$. And the lower limit widths of the $1 \mathrm{~s}$ and $1 \mathrm{p}$ states are $12 \pm 4 \mathrm{MeV}$ and $21 \pm 3 \mathrm{MeV}$, respectively, with $V_{0} \sim 15 \mathrm{MeV}$.

The binding energies of $K^{-}, B_{K}$, will decrease $1 \sim 2 \mathrm{MeV}$, if we increase the imaginary potential depths in their possible range. The widths do not affect the r.m.s. radii obviously.

If the imaginary potential depth $V_{0}$ is deeper than $50 \mathrm{MeV}$, the sum of the half widths of the $1 \mathrm{~s}$ and $1 \mathrm{p}$ states predicted by us for ${ }^{16} \mathrm{O} K^{-},{ }^{20} \mathrm{Ne} K^{-},{ }^{24} \mathrm{Mg} K^{-},{ }^{28} \mathrm{Si} K^{-},{ }^{32} \mathrm{~S} K^{-},{ }^{36} \mathrm{Ar} K^{-},{ }^{40} \mathrm{Ca} K^{-}$and ${ }^{44} \mathrm{Ti} K^{-}$are larger than the separations between the $1 \mathrm{~s}$ and $1 \mathrm{p}$ states for these kaonic nuclei, which implies that no discrete states can be identified in experiment for these nuclei. However, if $V_{0} \leq 30 \mathrm{MeV}$ the sum of the half widths of the $1 \mathrm{~s}$ and $1 \mathrm{p}$ states predicted by us are narrower than the separations of the two lowest energy levels, thus, the discrete $K^{-}$bound states should be identified in experiment.

The shrinkage effects for kaonic nuclei are found. The interior nucleon-densities do not increase drastically. The densest central nuclear density predict by us is about 2.1 times the normal nuclear density, however, which is much less than the value predicted in [19].

\section{Acknowledgements}

We would like to thank Prof. A. Gal for helpful discussions. This work was supported in part by the Natural Science Foundation of China (10275037, 10375074, 90203004) and China Doctoral Programme Foundation of Institution of Higher Education (20010055012).

[1] D. B. Kaplan and A. E. Nelson, Phys. Lett. B 175, 57 (1986).

[2] G. E. Brown and C. -H. Lee et al., Nucl. Phys. A567, 937 (1994).

[3] G. Q. Li, C. -H. Lee, and G.E. Brown, Nucl. Phys. A625, 372 (1997).

[4] J. Schaffner, I. N. Mishustin, and J. Bondorf, Nucl. Phys. A 625, 325 (1997).

[5] J. Schaffner, A. Gal, I.N. Mishustin, H. Stőcker, and W. Greiner, Phys. Lett. B 334, 268 (1994).

[6] J. Schaffner and I. N. Mishustin, Phys. Rev. C 53 (1996) 1416.

[7] T. Waas, N. Kaiser, and W. Weise, Phys. Lett. B 365, 12 (1996); Phys. Lett. B 379, 34 (1996); N. Kaiser, P. B. Siegel, and W. Weise, Nucl. Phys. A594, 325 (1995); W. Weise, ibid. A610, 35 (1996).

[8] T. Waas and W. Weise, Nucl. Phys. A625, 287 (1997).

[9] E. Oset, D. Cabrera, V.K. Magas, L. Roca, S.Sarkar, M.J. Vicente Vacas and A. Ramos, PRAMANA-journal of physics, 53, 1 (1999).

[10] S. Hirenzaki, Y. Okumura, H. Toki, E. Oset, and A. Ramos, Phys. Rev. C 61, 055205 (2000).

[11] A. Baca, C. García-Recio, J. Nieves, Nucl. Phys. A 673, 335 (2000).

[12] A. Cieplý, E. Friedman, A. Gal, J. Mareš, Nucl. Phys. A 696, 173 (2001).

[13] E. Friedman, A. Gal, C.J. Batty, Phys. Lett. B 308, 6 (1993); E. Friedman, A. Gal, C.J. Batty, Nucl. Phys. A 579, 518 (1994).

[14] E. Friedman, A. Gal, J. Mareš, A. Cieplý, Phys. Rev. C 60, 024314 (1999).

[15] E. Friedman and A. Gal, Phys. Lett. B 459, 43 (1999).

[16] Tadafumi Kishimoto, Phys. Rev. Lett. 83, 4701 (1999).

[17] M. Iwasaki, K. Itahashi, A. Miyajima, H. Outa, Y. Akaishi, T. Yamazaki, Nucl. Instru. Meth. Phys. Res. A 473, 286 (2001).

[18] T. Yamazaki and Y. Akaishi, Phys. Lett. B 535, 70 (2002).

[19] Y. Akaishi and T. Yamazaki, Phys. Rev. C 65, 044005 (2002).

[20] A. Doté, H. Horiuchi, Y. Akaishi, and T. Yamazaki, Phys. Rev. C 70, 044313 (2004).

[21] A. Doté, H. Horiuchi, Y. Akaishi, T. Yamazaki, Phys. Lett. B 590, 51 (2004).

[22] J. Mareš, E. Friedman, and A. Gal, Phys. Lett. B 606, 295 (2005).

[23] J. Mareš, E. Friedman, and A. Gal, nucl-th/0601009 
[24] N. Agnello, G. Beer , L. Benussi, et al., Nucl. Phys. A 752, 139c (2005).

[25] A. Dote, Y. Akaishi, T. Yamazaki, Nucl. Phys. A 754, 391c (2005).

[26] J. Yamagata , H. Nagahiro , Y. Okumura et al., Prog. Theor. Phys. 114, 301 (2005).

[27] E. Oset, H. Toki, nucl-th/0509048

[28] T. Suzuki, H. Bhang et al., Phys. Lett. B 597, 263 (2004).

[29] T. Suzuki, H. Bhang et al., Nucl. Phys. A 754, 375c (2005).

[30] T. Kishimoto, T. Hayakawa et al., Nucl. Phys. A 754, 383c (2005).

[31] M. Agnello, G. Beer et al. [FINUDA Collaboration], Phys. Rev. Lett. 94, 212303 (2005).

[32] V.K. Magas, E. Oset, A. Ramos and H. Toki, nucl-th/0601013

[33] R. Knorren, M. Prakash and P. J. Ellis, Phys. Rev. C 52,3470 (1995).

[34] X. H. Zhong, L. Li, C. H. Cai, and P.Z. Ning, Commun. Theor. Phys. 41, 573 (2004).

[35] R. Büttgen, K. Holinde, A. Müller-Groeling, J. Speth, and P. Wyborny, Nucl. Phys. A506, 586 (1990); A. Müller-Groeling, K. Holinde, and J. Speth, ibid., A513, 557 (1990).

[36] B. D. Serot and J. D. Walecka, Adv. Nucl. Phys. 16, 1 (1986).

[37] P.-G. Reinhard, Rep. Prog. Phys. 52, 439 (1989).

[38] M. M. Sharma and M. A. Nagaragian, Phys. Lett. B 312, 377 (1993).

[39] J. Yamagata, H. Nagahiro, Y. Okumura, and S. Hirenzaki, nucl-th/0503039

[40] K. Tanida et al., Phys. Rev. Lett. 86, 1982 (2001).

[41] Y. H. Tan, X. H. Zhong, C. H. Cai, and P. Z. Ning, Phys. Rev. C 70, 054306 (2004).

[42] X. H. Zhong, Y. H. Tan, G. X. Peng, L. Li, and P. Z. Ning, Phys. Rev. C 71, 015206 (2005).

[43] W. Scheinast et al., Phys. Rev. Lett. 96, 072301 (2006).

[44] K. Tsushima, D.H. Lu, A.W. Thomas and K. Saito, Phys. Lett. B 443, 26 (1998).

[45] A. Ramos, S. Hirenzaki, S.S. Kamalov , T.T.S. Kuo, Y. Okumura, E. Oset, A. Polls, H. Toki, L. Tolos, Nucl. Phys. A691, 258 (2001).

[46] C. Vander Velde-Wilquet, J. Sacton, J.H. Wickens, D.N. Tovee, D.H. Davis, Nuovo Cimento A 39, 538 (1977). 